\title{
The Impact of Prenatal Diagnosis of an Upper Extremity Anomaly on Pregnancy Outcome
}

\section{Matthew A Sullivan ${ }^{1}$ and Joshua M Adkinson ${ }^{2 \star}$}

${ }^{1}$ Northwestern University Feinberg School of Medicine, Indiana

${ }^{2}$ Department of Plastic Surgery, Riley Hospital for Children, University School of Medicine, Indiana

\begin{abstract}
Objective: Upper extremity anomalies (UEA) occur when there is an interruption in the normal process of embryonic limb development. The prenatal diagnosis of an UEA may impact the decision to deliver or terminate the pregnancy. We sought to elucidate factors associated with pregnancy outcome in the setting of a known UEA.
\end{abstract}

Methods: We reviewed electronic medical records at three affiliated hospitals to identify mothers with an UEA diagnosed by prenatal ultrasound. Maternal demographics, anomaly type, and clinical management variables were collected. Patients were stratified by primary outcome of either delivery or elective termination. Multivariate analysis was performed to identify factors associated with pregnancy termination.

Results: Thirty mother/child dyads were confirmed to have an UEA by prenatal ultrasound. Fourteen pregnancies resulted in live birth of the fetus $(47 \%)$ and 16 pregnancies were terminated $(53 \%)$. Pregnancies with multiple anomalies $(P<0.05)$ and/or bilateral UEAs $(P<0.05)$ were more likely to undergo termination. Consultation with an upper extremity surgeon was associated with delivery $(P<0.05)$. Maternal demographics were not associated with pregnancy outcome.

Conclusions: Fetal anomaly characteristics are associated with pregnancy outcome. Although prenatal consultation with an upper extremity surgeon was associated with delivery, this subset of patients were more likely to have isolated, unilateral anomalies.

Keywords: Upper extremity anomaly; Congenital hand differences; Abortion; Pregnancy termination; Prenatal ultrasound; Prenatal diagnosis

\section{Introduction}

An upper extremity anomaly (UEA) occurs when the natural development of the embryonic limb is altered due to environmental factors or genetic mutations [1]. While the prevalence of UEAs in the United States has yet to be thoroughly investigated, prevalence in other countries is estimated to occur in 3.4-21.5 per 10,000 live births [2-5].

Larger studies have found most UEAs to be isolated cases [3,4]. However, children with radial longitudinal deficiency, humeral defects, or bilateral reductions have a higher risk for associated anomalies and neonatal mortality $[4,5]$. Those with syndromic manifestations have higher rates of both neonatal mortality and spontaneous pregnancy termination [6].

The decision-making process related to pregnancy termination is extremely complex. The socioeconomic status, race, religion, age of the mother, as well as physician 'certainty' or 'recommendations', have been shown to significantly influence a mother's decision after discovering a congenital anomaly [7-14]. These decisions are further complicated in cases of UEAs, where underlying diagnoses and syndromic risks may be uncertain, and the perceived impact on quality of life is variable and multifactorial. Our goals are to provide clarity with regard to determinants of pregnancy termination after a prenatal diagnosis of an UEA and to determine whether there are variations in the rate of pregnancy termination based on type of UEA. For upper extremity surgeons, who rarely see mothers for prenatal counseling regarding a child with an UEA, this information will help facilitate a well-informed discussion.

\section{Materials and Methods}

After institutional review board approval, we performed a longitudinal analysis of medical records at three affiliated medical centers (Northwestern Memorial Hospital, Ann and Robert H. Lurie
Children's Hospital of Chicago, and Prentice Women's Hospital) for the years 2002 to 2015. We queried the prenatal ultrasound report database at Prentice Women's Hospital and Northwestern Memorial Hospital to identify mothers given a prenatal diagnosis of an UEA. The search parameters were developed by a Reproductive Geneticist with a thorough knowledge of the database. We reviewed the Northwestern Memorial Hospital Electronic Data Warehouse (EDW) to capture patients not included in the prenatal ultrasound database. As there are no discrete ICD-9 codes attributed to structural fetal anomalies of the limbs, we developed a hierarchical text search (Table 1) based on keywords as well as a query for all patients who underwent an elective pregnancy termination (ICD-9 codes: 635.xx, 636.xx, 637.xx, 638.xx) in combination with the search term "prenatal". Consultation notes in the electronic medical record (EMR) at Ann and Robert H. Lurie Children's Hospital were also included using the search parameters outlined in Table 1.

Mother/child dyads were included in the cohort when an UEA diagnosed via prenatal ultrasound was confirmed by review of the medical record. Mother/child dyads were excluded when further chart review revealed a false positive diagnosis, no definitive diagnosis, or skeletal dysplasia. For patients meeting inclusion criteria, we collected maternal demographics (age, race/ethnicity, gravida/para, marriage or equivalent status, socioeconomic status, characteristics of the UEA

*Corresponding author: Joshua M Adkinson, MD, Assistant Professor of Surgery, Division of Plastic Surgery, Riley Hospital for Children, Indiana University School of Medicine, 720 Eskanazi Ave, Indianapolis, IN 46202, Tel: 3179443636; E-mail: Joshadkinso@iu.edu

Received February 18, 2017; Accepted May 01, 2017; Published May 08, 2017

Citation: Sullivan MA, Adkinson JM (2017) The Impact of Prenatal Diagnosis of an Upper Extremity Anomaly on Pregnancy Outcome. Surgery Curr Res 7: 294. doi: 10.4172/2161-1076.1000294

Copyright: ( 2017 Sullivan MA, et al. This is an open-access article distributed under the terms of the Creative Commons Attribution License, which permits unrestricted use, distribution, and reproduction in any medium, provided the original author and source are credited. 


\begin{tabular}{|l|c|}
\hline Item 1 & "prenatal ultrasound" \\
\hline Item 2 & "upper limb" or "upper extremity" or "forearm" or \\
"arm" or "hand"
\end{tabular}

Table 1: ‘Electronic Data Warehouse' (EDW) text search

(type, unilateral vs. bilateral, date of detection, concomitant extremity anomalies), and maternal care clinical encounters (consultation with maternal fetal medicine [MFM], upper extremity surgeon, and/or other medical services). Socioeconomic status was extrapolated from the median household income by zip code, as reported by US Census Bureau data from the 2014 American Community Survey. Fetal UEA types observed in the present study were categorized into three main groups: hand anomalies (polydactyly, clinodactyly, cleft hand), limb deficiency (transverse, longitudinal), and other/complex anomalies (congenital contracture, micromelia, and limb body-wall complex (LBWC)).

Pregnancies were classified by the primary outcome of either delivery or elective termination. Statistical analysis was conducted to compare categorical data and to analyze demographics and clinical variables for our primary outcomes (delivery and termination) (SAS Software). Variables were dichotomized or reclassified into categories, when possible, to standardize the analysis and to achieve statistical power. P-values were calculated by chi-squared analysis and Fisher's exact test. Statistically significant differences in demographic and clinical data were calculated via Wilcoxon-Mann-Whitney analyses. Statistical significance was set at $\mathrm{P}<0.05$.

\section{Results}

We identified 981 mother/child dyads who met initial search criteria; 30 of which were confirmed to have an UEA by prenatal ultrasound (Figure 1). Fourteen pregnancies resulted in live birth of the fetus (47\%) and 16 pregnancies were terminated (53\%). Maternal demographics for each cohort are shown in Table 2. In univariate analysis, there were no statistically significant differences in maternal demographics between cohorts (Table 3). No significant differences in termination rates were found between the three categories of UEAs (hand anomalies (polydactyly, clinodactyly, cleft hand; $\mathrm{N}=11$ ), limb deficiencies (transverse, longitudinal; $\mathrm{N}=11$ ), and other/complex (constriction band, micromelia, LBWC; $\mathrm{N}=8$ ) (Table 4).

Those with multiple anomalies were more likely to undergo termination as compared to those with isolated anomalies $(\mathrm{P}<0.05)$. Bilateral UEAs were associated with a statistically significantly higher rate of termination as compared to isolated anomalies $(\mathrm{P}<0.05)$. Surgeon consultation was obtained in 55\% (6/11 patients) of isolated UEA cases versus $21 \%$ (4/19 patients) when associated with other anomalies. Consultation with an upper extremity surgeon was more likely to be obtained in the setting of an isolated UEA, but this did not reach statistical significance $(\mathrm{P}=0.06)$.

Consultation with an upper extremity surgeon was statistically significantly associated with delivery $(\mathrm{P}<0.05)$ (Table 5). Significant differences were also found when comparing median MFM encounters $(\mathrm{P}<0.05)$ and duration of care $(\mathrm{P}<0.05)$.

\section{Discussion}

The prenatal diagnosis of an UEA sets in motion a complex sequence of doctor-patient interactions and maternal decision-making

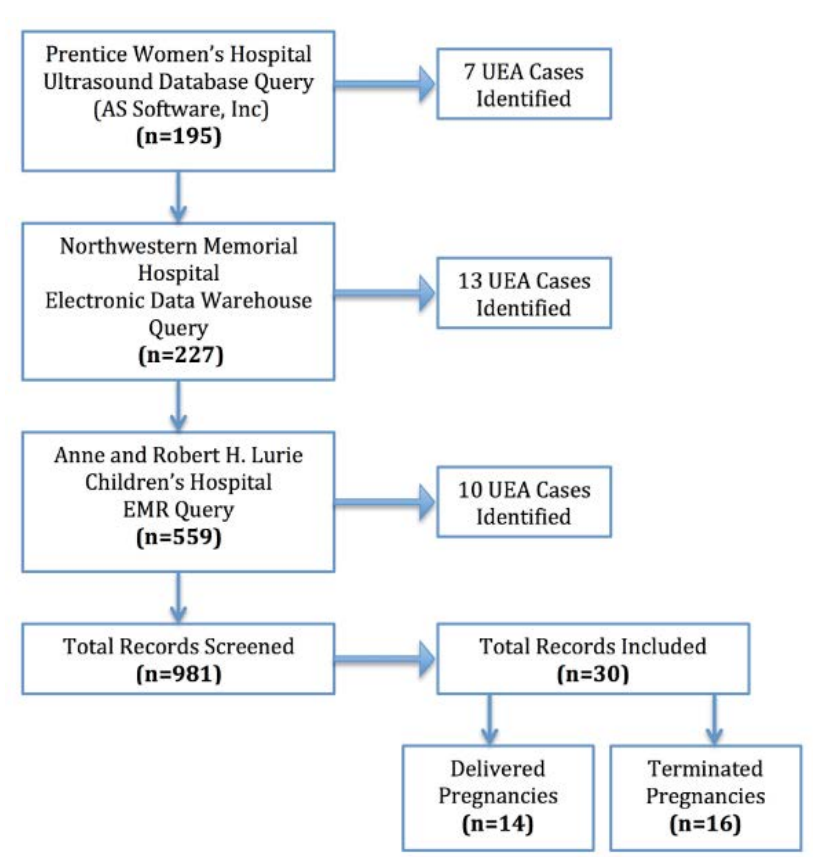

Figure 1: Cohort identification flowchart.

processes. We found several factors that are associated with pregnancy outcome. Mothers were significantly more likely to undergo elective pregnancy termination after diagnosis of bilateral UEAs. This decision may be influenced by the known association of bilateral UEAs with genetic trisomy or other syndromes linked to poor fetal outcomes [15]. Similarly, when the UEA was found in the setting of other non-upper extremity anomalies, pregnancy termination was more common. This aligns with previous studies showing that mothers are more likely to terminate a pregnancy in the setting of severe systemic anomalies [1619].

Our data indicate that prenatal consultation with an upper extremity surgeon is significantly associated with delivery. This is confounded, however, by the fact that surgeons evaluated a disproportionate number of patients with unilateral, isolated anomalies. Of the mothers that underwent prenatal surgical consultation $(n=10)$, the diagnosed fetal anomaly was more often unilateral (80\%, 8 of 10) and isolated $(60 \%, 6$ of 10$)$. These findings reflect the logical notion that referring obstetricians determine which children with prenatally diagnosed UEAs may theoretically benefit from future upper extremity reconstruction; patients with more complex or systemic anomalies are rarely, if ever, referred for evaluation. Many surgical options exist to improve both hand function and aesthetics in the setting of a variety of different congenital hand anomalies, though the impact on these outcomes depends heavily on the clinical phenotype and severity of the defect. We believe that greater access and referral to surgical counsel may improve outcomes for infants with remedial defects by allowing for surgical planning in the antepartum period or immediately after birth.

There were no significant associations between maternal sociodemographic variables and the ultimate decision to pursue pregnancy termination. This implies that the decision-making process is predominantly influenced by the UEA characteristics and associated anomalies, as well as MFM physician input. Other studies have shown that physician recommendations have a significant influence on maternal decisions regarding pregnancy termination [20]. Several other factors that may impact maternal decision-making were considered for 


\begin{tabular}{|c|c|c|}
\hline & Delivered Pregnancies & Terminated Pregnancies \\
\hline $\mathbf{N}$ & 14 & 16 \\
\hline \multicolumn{3}{|l|}{ Age (yrs) } \\
\hline Mean & 31.85 & 34.125 \\
\hline Range & $25-38$ & $24-40$ \\
\hline \multicolumn{3}{|c|}{ Race/Ethnicity } \\
\hline White & 4 & 11 \\
\hline Asian & 0 & 2 \\
\hline Black & 0 & 1 \\
\hline Hispanic & 3 & 0 \\
\hline Declined & 3 & 2 \\
\hline Unknown & 4 & 0 \\
\hline \multicolumn{3}{|c|}{ Marital Status } \\
\hline Married & 11 & 12 \\
\hline Single & 2 & 2 \\
\hline Divorced & 1 & 0 \\
\hline Unknown & 0 & 2 \\
\hline \multicolumn{3}{|l|}{ Insurance } \\
\hline Medicaid & 1 & 2 \\
\hline Private & 12 & 13 \\
\hline Self-Pay & 0 & 1 \\
\hline Unknown & 1 & 0 \\
\hline \multicolumn{3}{|c|}{ Household Income } \\
\hline$\$ 0-\$ 25 k$ & 0 & 0 \\
\hline$\$ 25-\$ 50 k$ & 5 & 3 \\
\hline$\$ 50-\$ 75 \mathrm{k}$ & 1 & 5 \\
\hline$\$ 75-\$ 100 k$ & 7 & 6 \\
\hline$\$ 100 k^{+}$ & 1 & 2 \\
\hline \multicolumn{3}{|l|}{ Gravida } \\
\hline 0 & 6 & 4 \\
\hline 1 & 3 & 5 \\
\hline$\geq 2$ & 5 & 7 \\
\hline \multicolumn{3}{|c|}{ Para (>37 wks) } \\
\hline 0 & 7 & 5 \\
\hline 1 & 4 & 5 \\
\hline$\geq 2$ & 3 & 6 \\
\hline \multicolumn{3}{|c|}{$\begin{array}{l}\text { Prior Preterm Births } \\
\text { (<37 wks) }\end{array}$} \\
\hline 0 & 12 & 14 \\
\hline$\geq 1$ & 0 & 2 \\
\hline \multicolumn{3}{|c|}{$\begin{array}{c}\text { Prior Abortions (<20 } \\
\text { wks) }\end{array}$} \\
\hline 0 & 10 & 12 \\
\hline$\geq 1$ & 2 & 4 \\
\hline \multicolumn{3}{|c|}{ Living Children } \\
\hline 0 & 7 & 5 \\
\hline 1 & 4 & 6 \\
\hline$\geq 2$ & 1 & 5 \\
\hline
\end{tabular}

inclusion in this study (i.e., social history, family history, environmental exposures, severe pregnancy complications), though this data was very rarely noted in the chart and was not sufficient for further analysis.

This study has several notable limitations. Our patient identification protocol was rigorous, but still limited by the lack of medical record integration between affiliated institutions. Without discrete ICD-9 codes for prenatally diagnosed structural fetal anomalies of the upper limbs, identification of our target population was imperfect. Despite efforts to maximize study inclusion, we were necessarily limited by dependence

\begin{tabular}{|c|c|c|c|}
\hline & $\begin{array}{l}\text { Delivered } \\
\text { Pregnancies } \\
\text { (N=14)a }\end{array}$ & $\begin{array}{c}\text { Terminated } \\
\text { Pregnancies } \\
(\mathrm{N}=16) \mathrm{a}\end{array}$ & P-value \\
\hline Maternal Age & & & \multirow{3}{*}{0.168} \\
\hline Median & 30.5 & 33.5 & \\
\hline Range & $25-38$ & $24-40$ & \\
\hline Maternal Race/ Ethnicity & & & \multirow{3}{*}{0.306} \\
\hline White & 4/7 (57.1\%) & 11/14 (78.6\%) & \\
\hline $\begin{array}{l}\text { Black, Hispanic, Asian, } \\
\text { Declined, Unknown }\end{array}$ & $3 / 7(42.9 \%)$ & $3 / 14(21.4 \%)$ & \\
\hline Marital Status & & & \multirow{3}{*}{0.564} \\
\hline Married & 11/14 (78.6\%) & $12 / 16(75.0 \%)$ & \\
\hline Single, Divorced, or Unknown & $3 / 14(21.4 \%)$ & $4 / 16(25.0 \%)$ & \\
\hline Insurance & & & \multirow{3}{*}{0.39} \\
\hline Private & $12 / 13(92.3 \%)$ & $13 / 16(81.3 \%)$ & \\
\hline Medicaid, Self-Pay & $1 / 13(7.7 \%)$ & 3/16 (18.8\%) & \\
\hline Household Income (\$) & & & \multirow{2}{*}{0.589} \\
\hline Median & 77,248 & 76,491 & \\
\hline Gravida & & & \multirow{4}{*}{0.576} \\
\hline 0 & 6/14 (42.9\%) & $4 / 16(25.0 \%)$ & \\
\hline 1 & 3/14 (21.4\%) & $5 / 16(31.3 \%)$ & \\
\hline$\geq 2$ & $5 / 14(35.7 \%)$ & 7/16 (43.8\%) & \\
\hline Para & & & \multirow{5}{*}{0.518} \\
\hline (term births >37 wks) & & & \\
\hline 0 & $7 / 14(50.0 \%)$ & $5 / 16(31.3 \%)$ & \\
\hline 1 & 4/14 (28.6\%) & $5 / 16(31.3 \%)$ & \\
\hline$\geq 2$ & $3 / 14(21.4 \%)$ & $6 / 16(37.5 \%)$ & \\
\hline Prior Preterm Births (<37 wks) & & & \multirow{3}{*}{0.204} \\
\hline 0 & $12 / 12(100.0 \%)$ & $14 / 16(87.5 \%)$ & \\
\hline$\geq 1$ & $0 / 12(0.0 \%)$ & $2 / 16(12.5 \%)$ & \\
\hline Prior Abortions (<20 wks) & & & \multirow{3}{*}{0.595} \\
\hline 0 & 10/12 (83.3\%) & $12 / 16(75.0 \%)$ & \\
\hline$\geq 1$ & 2/12 (16.7\%) & $4 / 16(25.0 \%)$ & \\
\hline Living Children & & & \multirow{4}{*}{0.236} \\
\hline 0 & $7 / 12(58.3 \%)$ & $5 / 16(31.3 \%)$ & \\
\hline 1 & 4/12 (33.3\%) & $6 / 16(37.5 \%)$ & \\
\hline$\geq 2$ & $1 / 12(8.3 \%)$ & $5 / 16(31.3 \%)$ & \\
\hline
\end{tabular}

a some $\mathrm{N}$ values lowered by exclusion of declined or unknown demographic information

Table 3: Univariate analysis of patient cohorts: delivery vs. termination.

on detection of UEAs by ultrasound as well as the heterogeneous documentation of prenatally diagnosed fetal anomalies. Although recognition of structural anomalies has increased in recent years with advancements in sonography and diagnostic protocols [21,22] detection rates in the United States are confounded by disparities in ultrasonographer expertise and the lack of national guidelines requiring documentation of anomalies in the distal extremities in low-risk pregnancies [23]. Because the management of complicated pregnancies may be distributed across different adult and pediatric health care systems, we may have lost patients to attrition. Further, the patients seen in our health care system may not be representative of those in other parts of the country; this is significant because of geographical variation in physician practice patterns as well as maternal beliefs regarding pregnancy termination. Lastly, our study cohort was small and this important factor limited the power of our statistical analysis.

Pregnancy termination due to a fetal anomaly has been associated with significant maternal emotional distress, perinatal grief, depression and post-traumatic stress disorder [24,25]. Asplin et al. found that such 


\begin{tabular}{|c|c|c|c|}
\hline & Delivered Pregnancies & $\begin{array}{l}\text { Terminated } \\
\text { Pregnancies }\end{array}$ & P-value \\
\hline Gestational Age at Dx & 145.43 & 133.67 & 0.498 \\
\hline \multicolumn{3}{|l|}{ Hand Anomalies } & \multirow{12}{*}{0.759} \\
\hline Polydactyly & $4 / 14(28.6 \%)$ & $4 / 16(25.0 \%)$ & \\
\hline Clinodactyly & $0 / 14(0 \%)$ & $1 / 16(6.3 \%)$ & \\
\hline Cleft Hand & $1 / 14(7.1 \%)$ & $1 / 16(6.3 \%)$ & \\
\hline Deficiency Anomalies & & & \\
\hline Transverse Deficiency & $5 / 14(35.7 \%)$ & $5 / 16(31.3 \%)$ & \\
\hline Longitudinal Deficiency & $1 / 14(7.1 \%)$ & $0 / 16(0 \%)$ & \\
\hline Other/Complex Anomalies & $\mathrm{t}$ & & \\
\hline Congenital Contracture & $1 / 14(7.1 \%)$ & $0 / 16(0 \%)$ & \\
\hline Micromelia & $1 / 14(7.1 \%)$ & $5 / 16(31.3 \%)$ & \\
\hline Limb Body Wall & \multirow{2}{*}{$1 / 14(7.1 \%)$} & \multirow{2}{*}{$0 / 16(0 \%)$} & \\
\hline Complex & & & \\
\hline Laterality & & & \multirow{3}{*}{$0.030^{*}$} \\
\hline Unilateral & $9 / 14(64.3 \%)$ & $4 / 16(25.0 \%)$ & \\
\hline Bilateral & $5 / 14(35.7 \%)$ & $12 / 16(75.0 \%)$ & \\
\hline \multicolumn{3}{|c|}{ Other Anomalies on Prenatal Ultrasound } & \multirow{3}{*}{$0.030^{*}$} \\
\hline 0 & $8 / 14(57.1 \%)$ & $3 / 16(18.8 \%)$ & \\
\hline$\geq 1$ (Non-UEAs) & $6 / 14(42.9 \%)$ & $13 / 16(81.3 \%)$ & \\
\hline
\end{tabular}

*statistically significant value $(P$-value $<0.05)$

Table 4: Comparison of fetal anomalies by outcome.

\begin{tabular}{|c|c|c|c|}
\hline & Delivered Pregnancies $(\mathrm{N}=14)$ & $\begin{array}{l}\text { Terminated Pregnancies } \\
\qquad(\mathrm{N}=16)\end{array}$ & P-value \\
\hline \multicolumn{3}{|l|}{ Upper Extremity Surgeon Consult } & \multirow{3}{*}{$0.001^{*}$} \\
\hline Obtained & $9 / 14(64.3 \%)$ & $1 / 16(6.3 \%)$ & \\
\hline Not obtained & $5 / 14(35.7 \%)$ & $15 / 16(93.8 \%)$ & \\
\hline Total MFM Encounters & 12.5 & 3.5 & $0.0033^{*}$ \\
\hline Median Duration of Care (days from diagnosis to delivery/termination) & 125.5 & 3.5 & $<0.0001^{*}$ \\
\hline
\end{tabular}

*statistically significant value $(P$-value $<0.05)$

Table 5: Categorical comparisons of maternal clinical data

mothers most often asked for changes in care related to "provision of adequate support through state-dependent communication, in-depth understanding and compassion.... and increased resources". As such, it is essential that the mother is well-informed regarding any realistic options for reconstruction and possible implications of the UEA on quality of life. This emotionally charged consultation should be driven by an understanding of the best available evidence with an appreciation for systemic conditions beyond the scope of care of the upper extremity surgeon $[13,14]$. Understanding the variables associated with pregnancy outcome in the setting of an UEA can lead to a more informed and useful prenatal consultation.

This study sought to identify differences between mothers who chose to deliver or to abort a complicated pregnancy to better understand factors that affects the decision making process. It must be clear that in all such cases, there is no preferred decision other than that chosen by the mother. The findings of this paper are intended to clinically facilitate an unbiased decision on behalf of the mother who is thoroughly informed on all possible treatments, therapies, and outcomes that exist after an upper extremity anomaly is diagnosed.

\section{Conclusion}

The arrangement of surgical treatment may be obtained when it can provide improved quality of life for those with less severe upper extremity anomalies. By contrast, termination may be selected when the prognosis is perceived as poor. Appropriate consultation with obstetricians, genetic counselors, and upper extremity surgeons can all play a critical role in the mother's decision-making process. Understanding these factors and the influence they have on the mother will facilitate better-coordinated care and more informed decisionmaking for parents facing this choice. Of the variables assessed in this study, severity of the upper extremity anomaly was the factor most predictive of the pregnancy outcome. Surgical consult should be considered in all cases of upper extremity anomaly diagnoses for a more completely informed decision-making process.

\section{Acknowledgements}

This research received no financial support or personal assistance from any funding agency in the public, commercial, or not-for-profit sectors.

\section{Statement of Ethics}

All procedures followed were in accordance with the ethical standards of the responsible committee on human experimentation (institutional and national) and with the Helsinki Declaration of 1975, as revised in 2008 (5).

The Ann and Robert H Lurie Children's Hospital of Chicago Institutional Review Board Panel \#2 (Lurie Children's IRB) reviewed and approved this study (Reference Number: IRB 2015-378) under risk/benefit determination as described in the Code of Federal Regulations (CFR) 45 Part 46, Subpart D: 45 CFR 46.404 Research not involving greater than minimal risk. This protocol was authorized by 45 Code of Federal Regulations (CFR) 46.111 and via expedited review as authorized by 45 CFR 46.110 the above-named protocol.

\section{Statement of Informed Consent}

The Lurie Children's IRB waives the requirement of obtaining informed consent 
Citation: Sullivan MA, Adkinson JM (2017) The Impact of Prenatal Diagnosis of an Upper Extremity Anomaly on Pregnancy Outcome. Surgery Curr Res 7: 294. doi: 10.4172/2161-1076.1000294

Page 5 of 5

for this study in accordance with 45 CFR 46.116(d): (1) the research involves no more than minimal risk to subjects; (2) the waiver or alteration will not adversely affect the rights and welfare of the subjects; (3) the research could not practicably be carried out without the waiver or alteration; (4) whenever appropriate, the subjects will be provided with additional pertinent information after participation.

The Lurie Children's IRB granted a complete (full) waiver of HIPAA authorization requirement under 45 CFR 164.512 (i) as the following criteria were satisfied: (1) The research could not be feasibly conducted without the waiver; (2) The research could not feasibly be conducted without access to the $\mathrm{PHI}$; (3) The use or disclosure of $\mathrm{PHI}$ involves no more than minimal risk to the subject's privacy and includes a plan to protect identifiers, a plan to destroy the identifiers at the earliest opportunity, and written assurances that the PHI will not be reused for purposes beyond what is described in the current study protocol.

\section{References}

1. Rayan GM, Upton J (2014) Congenital hand anomalies and associated syndromes. Springer, Verlag Berlin Heidelberg.

2. Giele H, Giele C, Bower C, Allison M (2001) The incidence and epidemiology of congenital upper limb anomalies: A total population study. J Hand Surg Am 26: $628-634$

3. Ekblom AG, Laurell T, Arner M (2010) Epidemiology of congenital upper limb anomalies in 562 children born in 1997 to 2007: A total population study from Stockholm, Sweden. J Hand Surg Am 35: 1742-1754.

4. Koskimies E, Lindfors N, Gissler M, Peltonen J, Nietosvaara Y (2011) Congenital upper limb deficiencies and associated malformations in Finland: A population-based study. J Hand Surg Am 36: 1058-1065.

5. Froster UG, Baird PA (1992) Upper limb deficiencies and associated malformations: A population-based study. Am J Med Genet 44: 767-781.

6. Paladini D, Greco E, Sglavo G, Armiento MR, Penner I, et al. (2010) Congenital anomalies of upper extremities: prenatal ultrasound diagnosis, significance, and outcome. Am J Obstet Gynecol 202: 596.

7. Smith LK, Budd JL, Field DJ, Draper ES (2011) Socioeconomic inequalities in outcome of pregnancy and neonatal mortality associated with congenital anomalies: Population based study. BMJ 343: 4306.

8. Schechtman K, Gray D, Baty J, Rothman SM (2002) Decision-making for termination of pregnancies with fetal anomalies: analysis of 53,000 pregnancies. Obstet Gynecol 99: 216-222.

9. Siffel C, Correa A, Cragan J, Alverson CJ (2004) Prenatal diagnosis, pregnancy terminations and prevalence of Down syndrome in Atlanta. Birth Defects Res Part A Clin Mol Teratol 70: 565-571.

10. Jackson JM, Crider KS, Cragan JD, Rasmussen SA, Olney RS (2014) Frequency of prenatal cytogenetic diagnosis and pregnancy outcomes by maternal race-ethnicity, and the effect on the prevalence of trisomy 21 , Metropolitan Atlanta, 1996-2005. Am J Med Genet A 164A: 70-76.

11. Zlotogora J, Haklai Z, Rotem N, Georgi M, Rubin L (2010) The impact of prenatal diagnosis and termination of pregnancy on the relative incidence of malformations at birth among Jews and Muslim Arabs in Israel. Isr Med Assoc J 12: 539-542.

12. Al-Matary A, Ali J (2014) Controversies and considerations regarding the termination of pregnancy for foetal anomalies in Islam. BMC Med Ethics 15: 10

13. Heuser CC, Eller AG, Byrne JL (2012) Survey of physicians' approach to severe fetal anomalies. J Med Ethics 38: 391-395.

14. Chervenak F, Mccullough LB (2012) Responsibly counselling women about the clinical management of pregnancies complicated by severe fetal anomalies. $J$ Med Ethics 38: 397-398

15. Pfeiffer RA, Santelmann R (1977) Limb anomalies in chromosomal aberrations Birth Defects Orig Artic Ser 13: 319-337.

16. Levi S, Hyjazi Y, Schaapst JP, Defoort P, Coulon R, et al. (1991) Sensitivity and specificity of routine antenatal screening for congenital anomalies by ultrasound: The Belgian multicentric study. Ultrasound Obstet Gynecol 1: 102110

17. Crane JP, LeFevre ML, Winborn RC, Evans JK, Ewigman BG, et al. (1994) A randomized trial of prenatal ultrasonographic screening: Impact on the detection, management, and outcome of anomalous fetuses. Am J Obstet Gynecol 171: 392-399.

18. Garne E, Loane M, Dolk H, De Vigan C, Scarano G, et al. (2005) Prenatal diagnosis of severe structural congenital malformations in Europe. Ultrasound Obstet Gynecol 25: 6-11.

19. Stoll C, Wiesel A, Queisser-Luft A, Froster U, Bianca S, et al. (2000) Evaluation of the prenatal diagnosis of limb reduction deficiencies. Prenat Diagn 20: 811 818

20. Gawron LM, Cameron KA, Phisuthikul A, Simon MA (2013) An exploration of women's reasons for termination timing in the setting of fetal abnormalities. Contraception 88: 109-115.

21. Chi LH, Stone DH, Gilmour WH (1995) Impact of prenatal screening and diagnosis on the epidemiology of structural congenital anomalies. J Med Screen 2: 67-70.

22. Salvador J, Borrell A, Lladonosa A (2005) Increasing detection rates of birth defects by prenatal ultrasound leading to apparent increasing prevalence. Lessons learned from the population-based registry of birth defects of Barcelona. Prenat Diagn 25: 991-996.

23. No authors listed (2013) AIUM Practice Guideline for the Performance of Obstetric Ultrasound Examinations. J Ultrasound Med 32: 1083-1101.

24. Asplin N, Wessel H, Marions L, Öhman SG (2014) Pregnancy termination due to fetal anomaly: Women's reactions, satisfaction and experiences of care. Midwifery 30: 620-627.

25. Korenromp MJ, Page-Christiaens GC, van den Bout J, Mulder EJ, Hunfeld JA, et al. (2005) Psychological consequences of termination of pregnancy for fetal anomaly: Similarities and differences between partners. Prenat Diagn 25: 1226-1233. 